\title{
BGP expression in gastric epithelium and early gastric cancer
}

\author{
Alfredo J.A. Barbosa and luiz P. Castro \\ Alfa Institute of Gastroenterology, University Hospital, Federal University of Minas Gerais, Av. Alfredo Balena $110,2{ }^{\circ} \mathrm{C}$ andar, $30.130-100$, \\ Belo Horizonte, Brazil
}

Gastric cancer continues to be, in many ways, one of the major challenges of modern gastroenterology. Significant progress was made in the last decades of the past century in terms of the early diagnosis and treatment of this neoplasia, with consequent progress in the clinical prognosis of the disease. However, many important features still need to be elucidated, among them those related to the evolution of early lesions from which gastric carcinoma originates. Particularly important today is the histopathological study of these lesions associated with immunohistochemistry, which represents one of the more attractive pathways for new advances that may further improve the progress obtained thus far in terms of modern practices of diagnosis and treatment, i.e., the understanding of precise methods of diagnosis for lesions of the gastric mucosa selectively associated with the genesis of gastric cancer. Thus, achieving relatively reliable methods for the diagnosis of precursor gastric cancer lesions that might be applied in a practical manner represents, today, a new frontier to be opened. We know that certain patterns of intestinal metaplasia of the gastric mucosa are strong candidates for precursor lesions of gastric cancer. Although there is no universal consensus, many investigators have proposed that incomplete type-III intestinal metaplasia with the presence of sulfomucin-producing columnar cells may be selectively linked to the development of gastric cancer [1,2]. On the other hand, we should also remember that the presence of intestinal metaplasia with sulfomucin-producing cells may simply represent an epiphenomenon linked to adaptive changes of the gastric mucosa debilitated by glandular atrophy and surrounded by an environment conditioned by the presence of multiple irritative and potentially carcinogenic factors. Topographic mapping of incomplete type-III intestinal metaplasia, even if it represents an epiphe-

Offprint requests to: A.J.A. Barbosa

Received: July 29, 2002 / Accepted: August 10, 2002 nomenon indicative of the presence of a risky microenvironment, might be of some use in practice if there should be a regular association of this type of metaplasia with gastric cancer, a finding that is not always, or indeed very seldom, observed [3]. Thus, the natural history of intestinal metaplasia in the gastric mucosa continues to be doubtful in terms of its specificity in relation to gastric carcinogenesis, even when reliable methods are used for the detection of sulfated mucins. From this viewpoint, intestinal metaplasia is considered by some not to be as strongly indicative of gastric cancer as is marked atrophy of the gastric mucosa itself [4].

In this issue of Gastric Cancer, Shimada and coworkers [5] again discuss this matter, emphasizing a possible immunohistochemical marker indicative of a high risk of development of gastric cancer, mainly applicable to patients submitted to treatment for an early carcinoma of the stomach. This study is a retrospective evaluation of the presence of brain (fetal)-type glycogen phosphorylase (BGP) in gastric mucosa with metaplasia, associated with different types of early gastric cancer. However, this is not their first report on the immunohistochemical detection of BGP in the metaplastic and neoplastic gastric epithelium. The same group of investigators has been studying the histopathology and the characteristics of immunoreactivity of anti-BGP antibodies in different situations. The first result of these studies dates back to 1984, when the group described the conspicuous expression of BGP in proliferative cells of intestinal metaplasia of the stomach and of well-differentiated gastric carcinoma [6]. At the time, the authors concluded that BGP expression and well-differentiated gastric adenocarcinoma may represent a more significant association than that occurring between intestinal metaplasia and gastric carcinoma. In summary, from a histological viewpoint, BGP expression by the gastric epithelium may provide a safer indication of carcinogenic potential of certain areas of the gastric epithelium. 
If, on the one hand, mucosectomy of early gastric carcinoma with no lymph node metastases offers an excellent prognosis to most patients, on the other hand, for a minority of patients (about $10 \%$ ), the final results are compromised: (1) by the existence of multiple, synchronous primary tumors not always detected precisely during the first intervention, (2) by tumor recurrence in the remaining stomach, an event that seems to be more frequent in patients presenting multiple tumors at first examination, and (3) by the presence of focal points of dysplasia in the gastric mucosa. High-grade dysplasia continues to represent a set of alterations of the gastric epithelium of which classification is still debated among different groups, being considered by some as the final point of preneoplastic changes, and by others as the starting point of gastric carcinoma. Furthermore, these lesions coexist with advanced carcinoma in most patients $[7,8]$. Regardless of the concept, dysplasia must be the result of a carcinogenic environment at the level of the gastric mucosa and must represent the major lesion responsible for the onset of intestinal-type carcinoma. However, in the metaplasia - dysplasia - carcinoma sequence, whatever the conceptual morphological limits between these different stages, the criterion at the basis of the point of no return of proliferative lesions is of fundamental importance when we are dealing with a patient surrounded by specialists in different areas and by different therapeutic options. Is the conspicuous, easily reproducible immunohistochemical expression of BGP in the gastric epithelium, regardless of the conceptual limits of precursor lesions of gastric carcinoma, a valid criterion for concrete medical attitudes towards the patient? We believe that the work of Shimada and co-workers takes a step in this direction, based on the experience and results obtained in the past few years. However, as the authors themselves emphasized, prospective studies with patient follow-up will be important in the future to validate the possible benefits of this laboratory methodology. If prospective studies should provide positive answers regarding the feasibility of this hypothesis, then the line of work of Shimada and co-workers will have, no doubt, implemented, in a significant manner, the clinical evaluation of patients with early gastric carcinoma with respect to the presence of a synchronous tumor or with respect to the extent of patient predisposition to metachronous carcinomas. In addition, it will have taken an important step towards rendering purely academic the conceptual discussions based on merely the morphological data of preneoplastic lesions of the gastric mucosa in relation to intestinal-type carcinoma.

\section{References}

1. Sipponen P, Seppala K, Varis K, Hjelt L, Ihamaki T, Kekki M, et al. Intestinal metaplasia with colonic-type sulphomucins in the gastric mucosa; its association with gastric carcinoma. Acta Pathol Microbiol Scand (A) 1980;88:217-24.

2. Segura DI, Montero C. Histochemical characterization of different types of intestinal metaplasia in gastric mucosa. Cancer 1983;52: 498-503.

3. Kato Y, Kitagawa T, Yanagisawa A, Kubo K, Utsude T, Hiratsuka $\mathrm{H}$, et al. Site-dependent development of complete and incomplete intestinal metaplasia types in the human stomach. Jpn J Cancer Res 1992;83:178-83.

4. El-Zimaity HM, Ramchatesingh J, Saeed MA, Graham DY. Gastric intestinal metaplasia: subtypes and natural history. J Clin Pathol 2001;54:679-83.

5. Shimada S, Shiomori K, Honmyo U, Maeno M, Yagi Y, Ogawa M. BGP expression in gastric biopsies may predict the development of new lesions after local treatment for early gastric cancer. Gastric Cancer 2002;5:130-6.

6. Shimada S, Maeno M, Misumi A, Akagi M. Histochemical study of phosphorylase in proliferating cells of intestinal metaplasia and carcinoma of the human stomach. Scand J Gastroenterol 1984;19: 965-70.

7. Lansdown M, Quirke P, Dixon MF, Axon ATR, Johnston D. High grade dysplasia of the gastric mucosa: a marker for gastric carcinoma. Gut 1990;31:977-83.

8. Kokkola A, Haapiainen R, Laxen F. Risk of gastric carcinoma in patients with mucosal dysplasia associated with atrophic gastritis: a follow-up study. J Clin Pathol 1996; 49:979-84. 